\title{
La evaluación de las bibliotecas de instituciones cubanas de investigación en biotecnología
}

\author{
Darlin Salgado-Batista*, Maria Victoria Guzmán-Sánchez*, \\ Yaidelyn Macías-Rivero*
}

\begin{abstract}
Resumen: La evaluación del desempeño de las Bibliotecas y su vínculo con el buen funcionamiento de las actividades de Investigación y Desarrollo (I+D) es incuestionable. Aunque los indicadores para la evaluación de bibliotecas han sido ampliamente abordados, se ha hecho poco para las bibliotecas vinculadas a los centros de alta tecnología, tales como los dedicados a la biotecnología. El objetivo de este trabajo es analizar la situación actual de la evaluación del desempeño en 22 bibliotecas pertenecientes a instituciones cubanas de investigación en biotecnología. Los elementos aquí presentados fueron recogidos a partir de la aplicación de una encuesta, en la cual se indagó sobre el uso y la importancia que le confiere el personal bibliotecario a este tipo de estudio. Los resultados de este trabajo pueden contribuir con el desarrollo de indicadores de desempeño para las bibliotecas pertenecientes a centros de I+D. Servirán, también, para enriquecer estudios comparativos entre diferentes tipos de bibliotecas.
\end{abstract}

Palabras claves: Evaluación de biblioteca, centros de investigación, Biotecnología, Cuba.

\section{The evaluation of libraries attached to Cuban institutions of biotechnology research}

\begin{abstract}
The evaluation of library performance and its relation to the effective functioning of R\&D activities is unquestionable. Although there has been broad coverage of indicators for library evaluation, little has been produced about libraries attached to centres of high technology, such as biotechnology. The aim of this paper is to analyse the current situation of performance evaluations in 22 libraries of Cuban biotechnology research centres. The data introduced were gathered from a survey inquiring in to the use by library staff of this kind of study and the importance attached to it. The outputs of this paper could contribute to the development of performance indicators for libraries belonging to R\&D centres. The outcome will also be useful for furthering comparative studies among different types of libraries.
\end{abstract}

Keywords: Library evaluation, research centre, biotechnology, Cuba.

\footnotetext{
* Centro de Investigaciones de los Alimentos, La Habana. Cuba. Correo-e: darlin@iiia.edu.cu. Recibido:13-04-2011, 2 . $^{\mathrm{a}}$ versión: 26-09-2011; 3. ${ }^{\mathrm{a}}$ versión: 16-12-2011; aceptado: 04-04-2012.
} 


\section{Introducción}

Las bibliotecas especializadas adscritas a centros de alta tecnología, como lo son los dedicados a la Biotecnología, están condicionadas por el dinamismo en la acumulación del conocimiento (McKelvey y otros, 2004). La estrategia en el manejo y gestión bibliotecaria en esta área científica, se ha tenido que enfrentar a un alto crecimiento de su producción documental (McKelvey, 2004). En este contexto, la evaluación bibliotecaria es obligatoria para buscar constantes alternativas de perfeccionamiento de los servicios y productos que se ofrecen.

Por todo lo anterior, el objetivo fundamental de este trabajo es analizar la situación actual de la evaluación del desempeño en 22 bibliotecas pertenecientes a instituciones cubanas de investigación en biotecnología.

Al personal que labora en estas bibliotecas se le aplicó una encuesta (anexo II) siguiendo el código deontológico la Sociedad Cubana de Ciencias de la Información (http://cultstgo.cult.cu/). Este estudio fue concebido como parte del proyecto "Propuesta y ensayo de un modelo híbrido para la evaluación de bibliotecas que pertenecen a centros de investigación biotecnológicos».

\section{Métodos y procedimientos}

El objeto de estudio estuvo formado por las bibliotecas pertenecientes a 22 instituciones (anexo I) del clúster biotecnológico de La Habana (López, 2006). El criterio de inclusión para conformar el universo fue el de aquellas bibliotecas adscritas a instituciones que usan la Biotecnología como parte de sus procesos de I+D. Se trabajó con todo el universo bajo estudio.

Se aclara que el clúster está integrado por instituciones que tienen dos niveles de agregación: un primer nivel para aquellas que están vinculadas directamente con la Biotecnología (10 instituciones). Un nivel dos para aquellas que usan proporcionalmente la biotecnología en un menor grado (12 instituciones). Del grupo uno, el $100 \%$ de la bibliotecas fueron encuestadas y el grupo dos el 75\% (9 de 12 instituciones). Se usaron como criterios de exclusión: (1) estar en proceso de re-estructuración organizacional, (2) estar mudándose de área física, (3) no recibir al encuestador.

Para el diseño y ajuste de la encuesta, se realizaron usando el método de análisis exploratorio, 8 entrevistas personales a bibliotecarios que laboran en estos centros. El objetivo de este pilotaje, fue conocer sobre los servicios y los indicadores de eficiencia por los que se les mide, tanto a la biblioteca como al personal que labora en ella.

La encuesta quedó configurada en 14 preguntas (anexo II), la primera parte tenía como objetivo recabar un conjunto de datos para caracterizar a las bibliotecas. La segunda, tenía entre otros propósito indagar sobre la existencia de los indicadores que fuesen medidos a las bibliotecas, así como el conocimiento que se tiene sobre los modelos de evaluación. En la encuesta se introducen los indi- 
cadores de visibilidad intra-institucional, su objetivo es valorar la importancia que se le confiere a la biblioteca dentro del centro de I+D.

\section{Resultados}

Es importante puntualizar que en este estudio se intenta indagar sobre el conjunto y no particularizar en cada biblioteca analizada. De los resultados del análisis estadístico descriptivo (agrupados según las tres dimensiones consideradas para el diseño de la encuesta: funcionalidad, evaluación y visibilidad), se pueden destacar los siguientes.

\subsection{Funcionalidad de las bibliotecas que conforman el clúster}

Todas las bibliotecas encuestadas, excepto un caso, ocupan la posición de departamentos, independientemente de la cantidad de personas que laboran, actividades que realizan o recursos tecnológicos que disponen. Menos homogéneos son los nombres de las áreas, estás fluctúan entre Gestión del Conocimiento y Gestión o Centro de Información. En este estudio se llamara, a cualquiera de estas unidades organizativas como bibliotecas.

El $74 \%$ de los encuestados eran especialistas o técnicos en Información Científica y Bibliotecología. Por lo que se evidencia una pertinencia entre la actividad y el puesto de trabajo.

La cantidad del personal varía entre 1 y 14 personas (tabla I), la mayor cifra se corresponde con aquellas bibliotecas que incluyen a la actividad editorial como una de sus tareas. Por ejemplo, la B9 tiene 14 personas y sus usuarios potenciales son 200. Se halló la media en función de la capacidad de servicio de cada biblioteca (razón usuario-personal). Los resultados aportaron que la media era de 199 personas atendidas por lo que solo 7 bibliotecas de las 19 encuestadas tenían una proporción óptima (B13, B11, B15, B14, B6, B12, B7, véase tabla I).

De las 19 bibliotecas encuestadas, 11 (56\%) eran híbridas, 4 (22\%) totalmente virtuales y 4 (22\%) tradicionales. Según los encuestados, a estas bibliotecas se les llama híbridas porque tienen servicios tradicionales y fondos en papel, pero, además, incluyen: fondos digitalizados, acceden y generan recursos virtuales como BDs, etc. A pesar de tener acceso a recursos digitales, 4 bibliotecas fueron clasificadas como tradicionales y no como híbridas. Lo anterior denota que en algunos casos existe confusión en definir el tipo de biblioteca.

Se identificó, además, que la no correspondencia entre cantidad de personal y tipo de biblioteca se debe a la diversidad de actividades que realizan y la misión de esa biblioteca dentro de la institución (respondida con las preguntas 13 y 14 anexo II). Incluso algunas bibliotecas aludieron que incluyen dentro de sus acciones la gestión de la propiedad intelectual, la elaboración de productos de valor añadido, así como la gestión de la información que se genera en otras áreas institucionales como las de producción o comercialización. 
TABLA I

Resumen de las categorias funcionales de las bibliotecas

\begin{tabular}{|c|c|c|c|c|c|}
\hline Bibliotecas & Personal & Usuarios & $\begin{array}{c}\text { Tipo } \\
\text { de biblioteca }\end{array}$ & Ordenadores & $\begin{array}{l}\text { Recursos } \\
\text { digitales }\end{array}$ \\
\hline B1 & 10 & 930 & Virtual & 22 & Sí \\
\hline B2 & 11 & 500 & Híbrida & 4 & Sí \\
\hline B3 & 4 & 250 & Híbrida & 4 & Sí \\
\hline B4 & 4 & 427 & Híbrida & 3 & Sí \\
\hline B5 & 2 & 200 & Híbrida & 4 & Sí \\
\hline B6 & 1 & 300 & Híbrida & 5 & Sí \\
\hline B7 & 1 & 200 & Híbrida & 2 & Sí \\
\hline B8 & 1 & 93 & Tradicional & 2 & Sí \\
\hline B9 & 14 & 200 & Hîbrida & 14 & Sí \\
\hline B10 & 5 & 180 & Tradicional & 5 & Sí \\
\hline B11 & 5 & 3.337 & Virtual & 15 & Sí \\
\hline B12 & 2 & 534 & Tradicional & 4 & Sí \\
\hline B13 & 1 & 675 & Híbrida & 1 & Sí \\
\hline B14 & 1 & 350 & Tradicional & 1 & No \\
\hline B15 & 2 & 773 & Hîbrida & 5 & Sí \\
\hline B16 & 6 & 900 & Virtual & 14 & Sí \\
\hline B17 & 5 & 70 & Virtual & 5 & Sí \\
\hline B18 & 5 & 920 & Hîbrida & 16 & Sí \\
\hline B19 & 2 & 98 & Híbrida & 3 & Sí \\
\hline
\end{tabular}

Se identificó que diferentes procesos de trabajo cotidiano requieren del acceso a todos los tipos y formas de información que se conservan en diferentes formatos y unidades organizativas dentro de la propia institución. En este sentido, el 33\% de las bibliotecas participan en proyectos para gestionar la información generada del proceso de producción y un $23 \%$ de actividades vinculada con recursos humanos o el control de la calidad.

Las 22 bibliotecas acceden, fundamentalmente, a servicios de revistas digitales usando las facilidades que ofrecen Hinari (http://hinari-gw.who.int/, EBSCO (www.ebscohost.com), PubMed Central (http://www.ncbi.nlm.nih.gov) y Scielo (http://www.scielo.org). Por otra parte, son utilizados los recursos de Infomed (www.sld.cu) (tabla II). Estas son las características fundamentales y comunes de las colecciones de títulos de revistas de las bibliotecas estudiadas.

En cuanto a la informatización de las bibliotecas y sus aportes en contenidos y servicios a su intranet institucional, solo el 53\% contribuyen a enriquecerla. 
TABLA II

Acceso a recursos online

\begin{tabular}{l|c}
\hline \multicolumn{1}{c|}{ Bases de datos } & $\begin{array}{c}\text { No de instituciones } \\
\text { que la usan }\end{array}$ \\
\hline INFOMED & 18 \\
\hline PubMed & 9 \\
\hline HINARI & 8 \\
\hline EBSCO & 5 \\
\hline Scielo & 5 \\
\hline
\end{tabular}

Estas bibliotecas son las que coinciden con aquellas que tienen mayor diversidad de actividades.

\subsection{Evaluación}

Las respuestas a las tres primeras preguntas de esta encuesta fueron fundamentales, pues se estableció que solo tres bibliotecas hacen evaluación bajo algún modelo lo que representa 16\%. El 37\% solo extrae determinadas estadísticas, mientras que el $47 \%$ no realiza ningún tipo de evaluación. Las bibliotecas que hacen algún tipo de evaluación lo hacen para los siguientes servicios: 1) Uso de recursos de información disponible en la intranet; 2) Encuesta a usuarios; 3) Préstamo de documentos; 4) Uso de BD y catálogos; 5) Accesos y otras estadísticas (biblioteca virtual); 6) Búsqueda bibliográfica, y 7) Reproducción de documentos.

El $47 \%$ que no realiza ningún tipo de evaluación, ante la pregunta ¿Por qué no se realiza? el 38\% de las personas respondieron que por desconocimiento de los modelos de evaluación y sus indicadores, también que no contaban con el personal suficiente. El 26\% no la realiza porque "la administración no lo exige» y otro 5\% considera que por "falta de tiempo". También se planteó "Los indicadores de evaluación no se aplican a nuestro tipo de biblioteca» (5\%).

A pesar de las respuestas anteriores, todos los encuestados aprecian la importancia que tiene la evaluación bibliotecaria y lo argumentan de forma coherente. Los motivos que argumentan son los siguientes: 1) Permite optimizar los recursos; 2) Conocer las necesidades de los usuarios y su percepción, ofrece la posibilidad de medir la efectividad e impacto de un servicio; 3) Identificar factores que facilitan el desarrollo del sistema de información; 4) Modo de retroalimentación para mejorar los servicios; 5) Identificar aportes y desventajas del sistema bibliotecario, así como medidas preventivas, y 6) Satisfacer las necesidades de los usuarios.

En la encuesta se les pidió a los especialistas, además, mencionar los modelos de evaluación bibliotecaria que conocían. 


\subsection{Modelos}

Es significativo que solo el $21 \%$ de los encuestados respondieron que conocían algún modelo de evaluación. Dos personas indicaron que conocía y había trabajado con la familia de normas o estándares de la «International Organization for Standardization» (ISO) y la "European Medicines Agency» (EMEA) establecidas para este tipo de industria.

A pesar que el $47 \%$ respondió que no realiza ningún tipo de evaluación, ante la pregunta: ¿Hace estudios de usuarios o estudios de necesidades? se comprobó que el 78\% respondió que sí lo realiza. Existía interés en indagar si los encuestados establecían a los estudios de necesidades o usuarios como diferentes a la evaluación bibliotecaria. Al calcular la diferencia entre el $47 \%$ y el $78 \%$, se pudo identificar que el 31\% los considera como procesos diferentes.

Las respuestas a la pregunta 6 (anexo II), permitió desglosar que los indicadores asociados al usuario (89\%) o la satisfacción de algún tipo de servicio o producto fue el tipo de indicador más extendido (tabla III).

\section{TABLA III}

\section{Estadísticas que se obtienen en las bibliotecas pertenecientes a un parque biotecnológico}

\begin{tabular}{c|l|c}
\hline $\mathbf{N}^{\mathbf{}}$ & \multicolumn{1}{|c}{ Indicador } & $\%$ \\
\hline 1 & Cantidad de usuarios que visitan o acceden a la biblioteca tradicional o virtual. & 73 \\
\hline 2 & Uso y satisfacción de los servicios ofertados (presencial o virtual). & 68 \\
\hline 3 & Nivel de satisfacción de los usuarios. & 68 \\
\hline 4 & Nivel de actualización del fondo (virtual o tradicional). & 63 \\
\hline 5 & Frecuencia de uso de las colecciones (presencial o virtual). & 63 \\
\hline 6 & Tiempo de respuesta en ofrecer un producto o servicio. & 63 \\
\hline 7 & Análisis de costo - beneficio. & 21 \\
\hline 8 & Posicionamiento en buscadores (en el caso virtual). & 16 \\
\hline 9 & Crecimiento del fondo (virtual o tradicional). & 5 \\
\hline 10 & Cadenas de búsquedas (temas) por los que acceden a la Biblioteca (virtual). & 5 \\
\hline 11 & Procedencia (países) de los usuarios que acceden a la Biblioteca (virtual). & 5 \\
\hline
\end{tabular}

Los indicadores 4 y 5 de la tabla III tratan sobre las colecciones de la biblioteca, mientras que el 6 implica medir el nivel de eficiencia con que trabaja el personal bibliotecario del centro. El análisis de estos datos permite deducir que el $100 \%$ de los bibliotecarios o especialistas en información reconocen que necesitan saber cómo funciona su sistema o parte de éste. Sin embargo, el 79\% desconoce los modelos de evaluación bibliotecarios y el $15 \%$ se rige para la 
evaluación por otras normas. Aún así, se extraen en conjunto 11 indicadores diferentes y se realizan estudios de usuarios.

\subsection{Indicadores de visibilidad intra-institucional}

El $68 \%$ de los encuestados tiene algún sistema que evalúa los resultados del personal bibliotecario. De estos, ocho indicadores de desempeño no están incluidos en los modelos de evaluación bibliotecaria como SERVQUAL (Butt, 2010). Los indicadores mencionados por los encuestados se agruparon en las siguientes categorías: 1) actividades extra-plan: realizan actividades fuera de las establecidas en su planificación o con un impacto para la institución; 2) nivel de aporte económico, y 3) Tiempo de ejecución (acortar plazos en la entrega de una tarea.

Este $68 \%$ tiene definido y evaluado por parte de la administración un conjunto de indicadores considerados de eficiencia económica-productiva. El personal bibliotecario está directamente implicado en los siguientes resultados: 1) participación en la gestión de la información generada a partir de los proceso u actividades de importancia institucional; 2) tipos de productos o servicios implicados directamente en la productividad; 3) participación en auditorías; 4) decisiones acertadas (toma de decisiones relacionadas con productos de valor añadido); 5) crecimiento productivo en ventas, y 6) participación en proyectos.

Es importante acotar que son considerados en el 58\% de los casos, también como resultados, las actividades relacionadas con la producción científica del bibliotecario (figura 1).

\section{FIGURA 1}

Indicadores medidos por la administración al personal bibliotecario

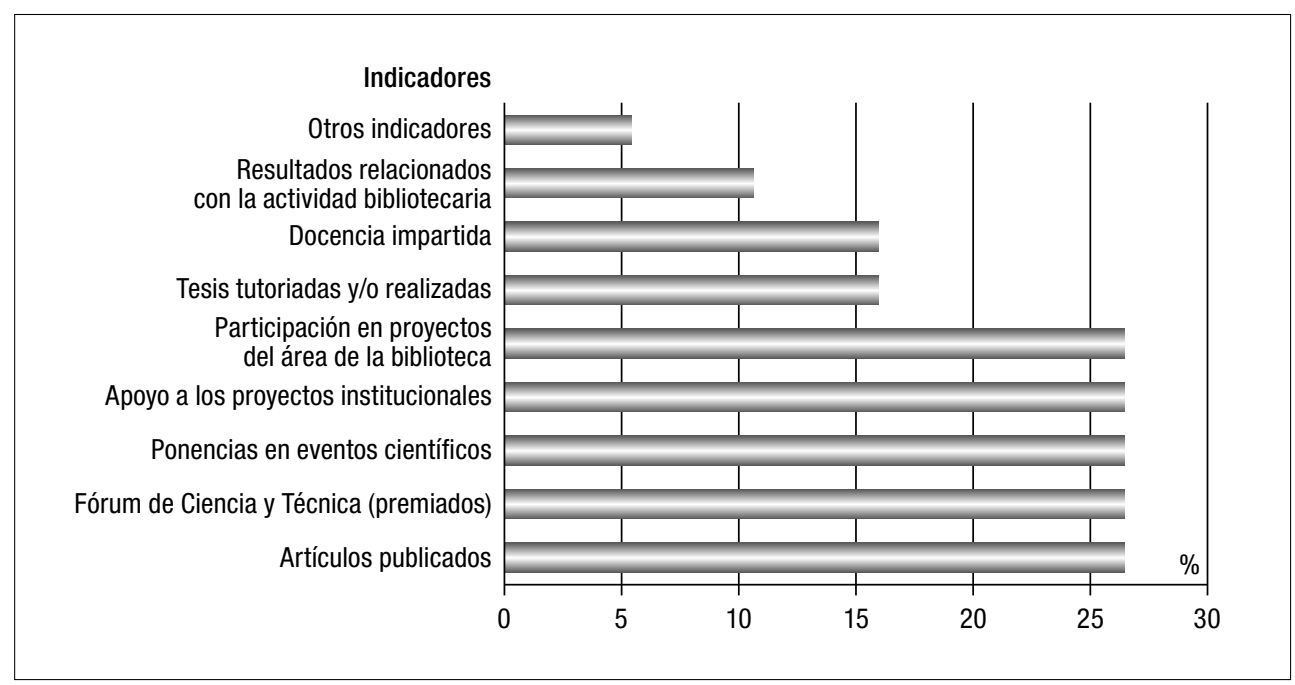




\section{Conclusiones}

Los resultados obtenidos en este estudio, permiten identificar que en las 22 bibliotecas encuestadas aún conviven todos los tipos de bibliotecas, aunque la tendencia más generalizada son las híbridas. Se aprecia la influencia de las tecnologías de la información y las comunicaciones (TICs), ya que cuatro de ellas son totalmente virtuales y tiene acceso (mayoritariamente) a colecciones virtuales. Por otra parte, no existe relación entre el tipo de biblioteca, la cantidad de personas dedicadas a las actividades bibliotecarias y los usuarios potenciales a atender. Sin embargo, sí se observa que las bibliotecas con mayor número de personal realizan mayor cantidad y diversidad de actividades.

Tal como se había previsto, los resultados demuestran que la totalidad de los bibliotecarios del clúster conocen de manera general la importancia de la evaluación bibliotecaria, así como de algunos indicadores que permiten indagar sobre las necesidades de los usuarios o servicios específicos (el 89\% realiza estudios de este tipo). Sin embargo, falta profundizar en los modelos que les permitiría tener una visión más general e integrar del proceso de evaluación.

Por lo que respecta a la existencia de algún sistema de evaluación para las bibliotecas por parte de los administrativos, resulta evidente que los indicadores de eficiencia económica-productiva tienen una preponderancia dentro del sistema de evaluación intra-institucional. En el $68 \%$ de los casos son evaluados bajo estos indicadores, mientras que ocho no están incluidos en los modelos de evaluación bibliotecaria conocidos.

Sin dudas, al ser la evaluación un proceso integrador y continuo donde se deben examinar los elementos principales de una organización así como la influencia de éstos en las condiciones interna y del entorno; se debe, extraer o definir un conjunto de indicadores que estén más acorde con el entorno de estas Bibliotecas. Como, por ejemplo, incluir los ocho indicadores que no aparecen en otros modelos. Igualmente, habría que contrastar los modelos e indicadores de evaluación bibliotecaria con otras especificaciones emitidas por entidades internacionales que tratan puntos referidos a la gestión de la información como la EMEA (2007). Esta podría ser una vía para ajustar los modelos de evaluación a las bibliotecas adscritas a centros biotecnológicos.

\section{Bibliografía}

Butt, M. M., y de Run, E. C. (2010): Private healthcare quality: applying a SERVQUAL model. Int J Health Care Qual Assur, vol. 23 (7), 658-73.

EMEA (2007): Records Management. European Union European Medicines Agency, EMA/590678/2007.

López M. E.; Silva, R.; Acevedo, B.; Buxadó, J. A.; Aguilera, A., y Herrera, L. (2006): Biotechnology in Cuba: 20 years of scientific, social and economic progress. Journal of Commercial Biotechnology, vol. 13, 1-11. 
McKelvey, M. D.; Rickne, A., y Laage-Hellman, J. (2004): The economic dynamics of modern biotechnology. UK: Edward Elgar Publishing, p. 418.

Rojas, J. L. (2001): Herramienta para la evaluación de los servicios de información en instituciones cubanas. La Habana: Universidad de La Habana.

\section{Anexo I. Centros visitados}

\begin{tabular}{|c|c|c|c|}
\hline $\mathbf{N} .^{\circ}$ & $\begin{array}{c}\text { Centros } \\
\text { encuestados }\end{array}$ & URL & Caracterización \\
\hline B1 & $\begin{array}{l}\text { Centro de Inmu- } \\
\text { nología Molecular } \\
(\mathrm{CIM}) \text {. }\end{array}$ & http://www.cim.co.cu/ & $\begin{array}{l}\text { Centro reconocido en la inmunoterapia del } \\
\text { cáncer, especialmente en el desarrollo de } \\
\text { «vacunas moleculares», ingeniería de anti- } \\
\text { cuerpos, ingeniería celular, bioinformática } \\
\text { y regulación de la respuesta inmune. }\end{array}$ \\
\hline B2 & $\begin{array}{l}\text { Instituto de Medi- } \\
\text { cina Tropical «Pe- } \\
\text { dro Kuri» (IPK). }\end{array}$ & http://www.ipk.sld.cu/ & $\begin{array}{l}\text { Es el máximo organismo de la vigilancia } \\
\text { epidemiológica en el país. Encargado de } \\
\text { proteger a la población de diversas enfer- } \\
\text { medades transmisibles (por ejemplo, el } \\
\text { SIDA). También trabaja en la I+D de vacu- } \\
\text { nas como la del dengue. }\end{array}$ \\
\hline B3 & $\begin{array}{l}\text { Centro de Sani- } \\
\text { dad Agropecua- } \\
\text { ria (CENSA). }\end{array}$ & http://www.censa.edu.cu/ & $\begin{array}{l}\text { Contribuye a preservar y elevar la sanidad } \\
\text { animal, vegetal y humana. Utiliza la bio- } \\
\text { tecnología en el mejoramiento de las es- } \\
\text { pecies y plantas. }\end{array}$ \\
\hline B4 & $\begin{array}{l}\text { Centro de Neuro- } \\
\text { ciencia. }\end{array}$ & $\begin{array}{l}\text { http://www.cneuro.co. } \\
\mathrm{cu} / ? \mathrm{q}=\mathrm{es} / \mathrm{cnch}\end{array}$ & $\begin{array}{l}\text { Se dedica a la investigación del cerebro } \\
\text { y la creación de tratamientos. }\end{array}$ \\
\hline B5 & $\begin{array}{l}\text { Centro de Inmu- } \\
\text { noensayo. }\end{array}$ & No tiene & $\begin{array}{l}\text { Es un centro insignia en el desarrollo y la } \\
\text { lucha contra diversas enfermedades (VIH, } \\
\text { dengue, chagas, etc.). También se especia- } \\
\text { liza en medicina para fetos. }\end{array}$ \\
\hline B6 & $\begin{array}{l}\text { Empresa Planta } \\
\text { de Sueros y Pro- } \\
\text { ductos Hemoderi- } \\
\text { vados. }\end{array}$ & No tiene & $\begin{array}{l}\text { Se dedica a producir con calidad y efi- } \\
\text { ciencia para uso humano, parenterales de } \\
\text { gran volumen, soluciones concentradas } \\
\text { para hemodiálisis y hemoderivados. }\end{array}$ \\
\hline B7 & $\begin{array}{l}\text { Instituto de Inves- } \\
\text { tigaciones para la } \\
\text { Industria Alimenti- } \\
\text { cia (IIIA). }\end{array}$ & $\begin{array}{l}\text { http://www.cuba.cu/inves- } \\
\text { tigaciones_alimentos/de- } \\
\text { fault.htm }\end{array}$ & $\begin{array}{l}\text { Encaminados a contribuir al desarrollo de } \\
\text { la industria procesadora de alimento y } \\
\text { bebidas y a la satisfacción de las necesi- } \\
\text { dades alimentarias y nutricionales de la } \\
\text { población cubana. Utiliza la Biotecnología } \\
\text { aplicada al desarrollo de alimentos. }\end{array}$ \\
\hline B8 & $\begin{array}{l}\text { Centro Nacional } \\
\text { Genética Médica. }\end{array}$ & $\begin{array}{l}\text { http://www.sld.cu/sitios/ } \\
\text { genetica/verpost.php?blog } \\
\text { =http://articulos.sld.cu/ } \\
\text { genetica\&post_id=195\&c= } \\
2987 \& \text { tipo }=2 \& \text { idblog }= \\
\text { 141\&p=1\&n=de }\end{array}$ & $\begin{array}{l}\text { Centro de referencia nacional para el Pro- } \\
\text { grama Cubano de Diagnóstico Manejo y } \\
\text { Prevención de Enfermedades Genéticas y } \\
\text { Defectos Congénitos. }\end{array}$ \\
\hline
\end{tabular}




\begin{tabular}{|c|c|c|c|}
\hline N. ${ }^{\circ}$ & $\begin{array}{c}\text { Centros } \\
\text { encuestados }\end{array}$ & URL & Caracterización \\
\hline B9 & $\begin{array}{l}\text { Centro Nacional } \\
\text { de Investigacio- } \\
\text { nes Científicas } \\
\text { (CENIC). }\end{array}$ & http://www.cnic.edu.cu/ & $\begin{array}{l}\text { Se dedicada a la investigación farmacéuti- } \\
\text { ca y biotecnológica. Produce medicamen- } \\
\text { tos, equipamiento y materiales biocompa- } \\
\text { tibles. También está desarrollando } \\
\text { nanotecnología e investigando con ozono. }\end{array}$ \\
\hline B10 & $\begin{array}{l}\text { Centro de Quími- } \\
\text { ca Biomolecular } \\
\text { (C.Q.B). }\end{array}$ & No tiene & $\begin{array}{l}\text { Trabaja en el desarrollo a ciclo completo } \\
\text { de biomoléculas, utilizando herramientas } \\
\text { de la química para la obtención de vacu- } \\
\text { nas y productos similares, fundamental- } \\
\text { mente para la salud humana. }\end{array}$ \\
\hline B11 & $\begin{array}{l}\text { Centro de Investi- } \\
\text { gación y Desarro- } \\
\text { llo de Medica- } \\
\text { mentos (CIDEM). }\end{array}$ & $\begin{array}{l}\text { http://www.cubanhealth. } \\
\text { com/centros_instituciones/ } \\
\text { cidem.htm }\end{array}$ & $\begin{array}{l}\text { Investiga sobre productos naturales y su } \\
\text { aplicación en la industria farmacéutica. } \\
\text { Desarrolla tecnologías para obtener me- } \\
\text { dicamentos terminados. }\end{array}$ \\
\hline B12 & $\begin{array}{l}\text { Centro para la } \\
\text { Producción de } \\
\text { Animales de La- } \\
\text { b o r a t o r i o s } \\
\text { (CENPALAB). }\end{array}$ & $\begin{array}{l}\text { http://www.ecured.cu/in- } \\
\text { dex.php/Centro_Nacional_ } \\
\text { para_la_Producci\%C3\% } \\
\text { B3n_de_Animales_de_La- } \\
\text { boratorio }\end{array}$ & $\begin{array}{l}\text { Produce y comercializa especies y razas } \\
\text { de laboratorio, domésticas, productivas } \\
\text { y exóticas, así como biopreparados, uti- } \\
\text { lizable como materia prima fundamental } \\
\text { en la elaboración de vacunas contra la } \\
\text { hepatitis B y el cáncer. }\end{array}$ \\
\hline B13 & Laboratorio AICA. & $\begin{array}{l}\text { http://www.quiminet.com/ } \\
\text { sh5/sh_advcrsdfadvcaass. } \\
\text { htm }\end{array}$ & $\begin{array}{l}\text { Se encarga de la elaboración de medica- } \\
\text { mentos para uso humano. }\end{array}$ \\
\hline B14 & $\begin{array}{l}\text { Instituto de Inves- } \\
\text { tigaciones Farma- } \\
\text { céuticas (IFAL). }\end{array}$ & http://www.ifal.uh.cu/ & $\begin{array}{l}\text { Institución encargada de la formación. De- } \\
\text { sarrolla investigaciones científicas vincula- } \\
\text { das a importantes aspectos del desarrollo } \\
\text { científico, técnico, económico y social. }\end{array}$ \\
\hline B15 & $\begin{array}{l}\text { Hospital Ortopé- } \\
\text { dico «Frank País». }\end{array}$ & $\begin{array}{l}\text { http://www.ecured.cu/in- } \\
\text { dex.php/Hospital_Or- } \\
\text { top\%C3\%A9dico_Frank_ } \\
\text { Pa\%C3\%ADs }\end{array}$ & $\begin{array}{l}\text { Brinda atención especializada en ortope- } \\
\text { dia, incluyendo tumores óseos, trastornos } \\
\text { neurológicos y osteomiarticulares. }\end{array}$ \\
\hline B16 & Instituto Finlay. & http://www.finlay.sld.cu/ & $\begin{array}{l}\text { Se dedica a la I+D y producción de va- } \\
\text { cunas. Así como la investigación básica } \\
\text { de enfermedades infecciosas. }\end{array}$ \\
\hline B17 & $\begin{array}{l}\text { Centro de Ci- } \\
\text { bernética Aplica- } \\
\text { da a la Medicina. } \\
\text { (CECAM). }\end{array}$ & www.cecam.sld.cu/ & $\begin{array}{l}\text { Centro rector para la enseñanza de la In- } \\
\text { formática Médica en el pregrado de todas } \\
\text { las carreras de la enseñanza médica supe- } \\
\text { rior. Especializado en bioinformática, y } \\
\text { modelaje informático de moléculas. }\end{array}$ \\
\hline B18 & $\begin{array}{l}\text { Centro de Inge- } \\
\text { niería Genética y } \\
\text { Biotecnología } \\
\text { (CIGB). }\end{array}$ & http://www.cigb.edu.cu/ & $\begin{array}{l}\text { Se especializa en productos obtenidos por } \\
\text { ingeniería genética: Heberprot-P (medica- } \\
\text { mento para el pie diabético), vacunas te- } \\
\text { travalente y pentavalente (en colabora- } \\
\text { ción con el Instituto Finlay), e interferones. }\end{array}$ \\
\hline
\end{tabular}




\begin{tabular}{c|l|c|l}
\hline $\mathbf{N}^{\circ}$ & $\begin{array}{c}\text { Centros } \\
\text { encuestados }\end{array}$ & \multicolumn{1}{c}{ URL } & \multicolumn{1}{c}{ Caracterización } \\
\hline B19 & $\begin{array}{l}\text { Centro Nacional } \\
\text { Coordinador de } \\
\text { Ensayos Clínicos } \\
\text { (CENCEC). }\end{array}$ & http://www.cencec.sld.cu/ & $\begin{array}{l}\text { Centro dedicado a garantizar la evaluación } \\
\text { línica que se requiere para el registro y la } \\
\text { comercialización de productos médico-far- } \\
\text { macéuticos o biotecnológicos. Además, rea- } \\
\text { liza evaluaciones terapéuticas para solucio- } \\
\text { nar los problemas de salud de la población. }\end{array}$ \\
\hline $\begin{array}{l}\text { Centro de Histote- } \\
\text { rapia Placentaria. }\end{array}$ & $\begin{array}{l}\text { Http://www.histoterapia- } \\
\text { placentaria.cu/ }\end{array}$ & $\begin{array}{l}\text { Se dedicada a la investigación y produc- } \\
\text { ción de medicamentos, cosméticos, diag- } \\
\text { nosticadores y nutrientes derivados de la } \\
\text { placenta humana }\end{array}$ \\
\hline B21 & $\begin{array}{l}\text { Centro Nacional } \\
\text { de Biopreparados } \\
\text { (BIOCEN). }\end{array}$ & $\begin{array}{l}\text { Producen y desarrollan parenterales de } \\
\text { bajo volumen, liofilizados, vacunas y otros } \\
\text { productos biotecnológicos. Además, in- } \\
\text { vestigan sobre medios de cultivo y pro- } \\
\text { ductos afines, antianémicos y productos } \\
\text { para el diagnóstico e inmunoterapia de } \\
\text { las alergias. }\end{array}$ \\
\hline
\end{tabular}

Nota: Los centros relacionados no están organizados en función del orden dado en la tabla I.

\section{Anexo II. Encuesta}

El motivo de esta encuesta es recabar elementos que nos ayuden a confeccionar un modelo de evaluación de las bibliotecas científicas, adscritas a centros de investigación biotecnológicos.

Este modelo debe incluir los típicos indicadores de evaluación pero también nuevos indicadores como los implementados en la resolución 9 (pago por resultados). El modelo también debe servir para bibliotecas digitales como tradicionales.

De antemano, le agradecemos su cooperación en esta investigación.

\section{Generalidades de la Organización}

Nombres y apellidos:

Profesión:

Cargo que ocupa:

Grado científico:

Centro del Polo al que pertenece:

Cantidad de trabajadores vinculados a la unidad de información:

Nivel de esos profesionales:

$$
\text { Dr.C.:___ MsC.:___ Lic.:___ Técnicos: }
$$


¿Cómo usted considera la biblioteca a la que pertenece?

$\begin{array}{ll}\text { Tradicional } \\ \square & \text { Digital } \\ & \text { Electrónica } \\ & \text { Virtual } \\ & \text { Híbrida } \\ & \text { Otra }\end{array}$

¿Por qué?

Cantidad de computadoras en su unidad:

Acceso a Internet Sí No

Acceso a los recursos: E.mail Infomed Otros

Acceso a Bases de datos: Internas Nacionales Internacionales ¿Cuáles?

Acceso a suscripciones digitales: De pago Gratuitas Ambas

\section{Revistas online} Boletines

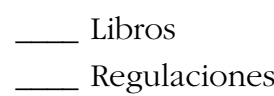

¿Qué servicios automatizados desarrollan en su biblioteca?:

Fondo digitalizado Revistas Open Access Otros

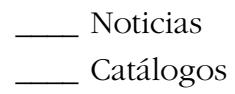

Catálogos
Boletín electrónico BD

¿Cuáles?

Cantidad de usuarios Potenciales/reales:

\section{Preguntas}

1. Se realiza evaluación de los servicios bibliotecarios:

$$
\text { _ Todos_ Algunos }
$$
No se realiza

Mencione a qué servicio le realiza evaluaciones

2. En caso de no realizarse la evaluación, ¿por qué no se hace?

__ Desconocimiento de los indicadores de evaluación bibliotecaria. La administración no la exige.

Los indicadores de evaluación no se aplican a nuestro tipo de bibliotecas.

_ No lo creemos necesario.

_ No contamos con el personal adecuado para realizarlo.

_ Otras, ¿Cuáles? 
3. ¿Qué importancia tiene para usted la evaluación bibliotecaria?

4. ¿Conoce algún modelo de evaluación bibliotecaria?

¿Cuáles?

1.

5. ¿Hace estudios de usuarios o estudios de necesidades?

Sí, frecuencia _ No

6. ¿Lleva algún indicador o estadística en la bibliotecas? Especifique:

Actualización del fondo (virtual o tradicional).

Frecuencia de uso de las colecciones (presencial o virtual).

Visitas (biblioteca tradicional o virtual).

__ Uso y satisfacción de los servicios ofertados (presencial o virtual).

Posicionamiento en buscadores (en el caso virtual).

Análisis de costo - beneficio.

Tiempo de respuesta en ofrecer un producto o servicio.

Nivel de satisfacción de los usuarios.

Otros. Cuáles:

\section{Preguntas relacionadas con la estimulación}

7. ¿Tiene la biblioteca algún sistema de estimulación (pago por resultados, resolución, etc.?

¿Cuáles?

8. Nivel o categoría que tiene la biblioteca dentro de este sistema.

9. ¿Bajo qué indicadores de desempeño le miden?

1.

2

3.
4.

5 .

6.

10. ¿Se han definido indicadores de eficacia y de eficiencia de los procesos bibliotecarios? $\begin{array}{lll}\text { ¿Cuáles?: } & \text { Sí } & \text { No } \\ 1 . \longrightarrow & 3 . \\ 2 . & 4 .\end{array}$ 
11. ¿Se cobra algún producto o s ervicio?

¿Cuáles?:

1.

3.

2 .

4.

12. ¿Se llevan los costes y gastos de la biblioteca?

$\begin{array}{lll}\text { ¿Cuáles?: } & \text { Sí } & \text { No } \\ 1 . \longleftarrow & 3 . \\ 2 . \longleftarrow & 4 .\end{array}$

13. ¿Tipo de servicios que ofrece?

14. Se les paga como parte del estímulo:

Artículos publicados.

Participación con ponencias en eventos científicos.

Trabajo presentado en el Forum de Ciencia y Técnica.

Apoyo a los proyectos o investigaciones de otras áreas.

Resultados sobresalientes relacionados con la actividad bibliotecaria.

Participación en proyectos del área de la biblioteca.

Tesis dirigidas y/o realizadas.

Docencia impartida.

Otros indicadores.

¿Cuáles?

1.

3

2

4

\section{Muchas gracias por su cooperación.}

Nota: Usted recibirá un ejemplar del modelo de evaluación en cuanto esté concluido el estudio. 